\title{
Towards an Internet of Things for Health Centers in the Southern Saudi Arabia Region
}

\author{
Mohamed Nadhmi Miladi ${ }^{1 *}$, Abdel Rahman Hasan ${ }^{2}$, Mohamed Al Ghobiri ${ }^{3}$, Osman Nasr ${ }^{4}$, and \\ Ahmed Abelmotlab ${ }^{5}$ \\ ${ }^{1-5}$ Department of Management Information Systems, Business College, King Khaled University \\ Guraiger, Abha 62529, Saudi Arabia \\ Email: ${ }^{1}$ mmiladi@kku.edu.sa, ${ }^{2}$ abhamohamed@kku.edu.sa, ${ }^{3}$ maalghobiri@kku.edu.sa, \\ 4 oanassr@kku.edu.sa, ${ }^{5}$ aabdelmotlab@kku.edu.sa
}

\begin{abstract}
The health centers in the village are well equipped in terms of infrastructure. It also has a large group of patients and staff who usually run services such as opening the file and adding data of patients or doctors using the papers and searching between the patient files. This manual job is challenging to do and wastes time. The developed system in the research aims to transform the center into a technology-based center by leaving paperwork in their daily work. The proposed system is considered as the first part of a global and integrated solution based on Internet of Things (IoT) technology. It seeks the enhancement of the Saudi Arabia health system, especially in the current pandemic period. The whole analysis diagrams are realized through the Visual Paradigm tool suite. The patient file management system has three types of modules, including the administrator, doctor, and receptionist. The proposed system provides a computerized patient file management in the health center. It will benefit the small health center and the receptionists who currently depend on the manual system. The proposed system is automated patient file management by integrating hospital information and details and healthcare information of the patients to a central system database. It also provides receptionists an easy way for booking an appointment according to patients' preference, and they can search and access patients' information conveniently.
\end{abstract}

Index Terms-Internet of Things (IoT), Health Centers, Southern Saudi Arabia

\section{INTRODUCTION}

$\mathbf{N}$ OWADAYS, health is more vital than before. People's health, especially in a pandemic phase, drives major government sectors, such as the economy and finance [1]. Likewise, in the last decade, technological advances and innovations offer solutions and perspectives that are unreachable before. Internet of Things (IoT) can change lifestyle in the coming

Received: Oct. 21, 2020; received in revised form: Jan. 23, 2021; accepted: Jan. 25, 2021; available online: March 04, 2021. *Corresponding Author years [2]. Taking advantage of such technologies with health requirements becomes a huge challenge [3]. It addresses the health problem in the southern Saudi Arabia region regarding IoT technologies [4].

The health centers in the village are well equipped in terms of infrastructure. It also has a large group of patients and staff who usually run services such as opening the file and adding data of patients or doctors using the papers and searching between the patient files. These manual works are hard to do and waste time. If people rely on the paper system, they may lose the paperwork or damage the papers. Many complaints have been made about the loss of patient files and the difficulties associated with transferring files to the hospitals and alternative clinics. From time to time, the staff hears complaints about the loss of medical files or the delay due to the searching process and the finding of medical files for a long time and congestion during the morning. Obviously, medical files have become a problem. However, the loss of a medical file is also a concern for the patients. Hence, a solution is needed to move the file without papers and exploit the information that penetrates lives and becomes an integral part. Hospitals have emptied a range of traditional medical records, files, radiographs, and other documents relating to patients and medical history in many developed countries. These files have been transferred to electronic records and documents that are updated and developed according to patients' frequency, changes, and developments. Several previous researchers [5-8] propose solutions to tackle the problem of health systems. However, these solutions fail to handle the specific characteristics of Saudi Arabia in general and the southern region specifically.

The research aims to develop the center by introducing technology in their work. Thus, it will facilitate the task of managing the center in the right way. It 
Cite this article as: M. N. Miladi, A. R. Hasan, M. A. Ghobiri, O. Nasr, and A. Abelmotlab, "Towards an Internet of Things for Health Centers in the Southern Saudi Arabia Region", CommIT (Communication \& Information Technology) Journal 15(1), 1-7, 2021.

is expected to transform the center into a technologybased center by leaving paperwork in their daily work to develop the system and general function. It enhances the previous works by overcoming their shortness and provides a dedicated solution for health services in the southern Saudi Arabia region.

\section{LiterATURE REVIEW}

\section{A. Previous Research}

The previous researchers propose a unique cloudbased hospital management system for both patients and hospital staff (doctors and management). The primary target of this design is to make people have a better experience in the hospital than the current one. Hospital is a place where no one willingly wants to visit, but there are times when people need to. The old system of hospitals is also not very user friendly. There are lots of problems that make hospital experience terrible. For example, the patients wait in the queue for long hours. The main focus is to make people's lives easier in the hour of need. The system will reduce much paperwork and save time [9].

Another previous research provides a design and implementation of the hospital management system. Before a computerized hospital management system comes into practice, it is difficult to keep proper records of the daily activities of hospitals, patient information, equipment maintenance schedule in the hospital, and how funds are allocated and used. It results in a waste of money, time, and workforce. The hospital management system is the designed information management system to manage the various aspects of the hospital (administrative, clinical, and financial issues). It helps to monitor and control the hospital's daily transactions and performance. It also addresses the critical requirements in the hospital. The system enables access to the right information and automation of complex tasks. Thereby, it allows the staff to spend more time caring for patients. The system is custom built to meet the specific requirements of medium and large size hospitals across the globe [5].

Next, another research designs a hospital management system that deals with the patients' health. Good medical care relies on well-trained doctors and nurses and high-quality facilities and equipment. It also relies on good record keeping. Without accurate, comprehensive, up-to-date, and accessible patient notes, medical personnel may not offer the best treatment or may misdiagnose the condition, which can have serious consequences. Associated records, such as x-rays, specimens, drug records, and patient registers, must also be well cared for. Good records also ensure several things. First, the administration in the hospital runs smoothly. Second, the unneeded records are transferred or destroyed regularly. Third, it keeps storage areas clear and accessible. Fourth, essential records can be found quickly. Hence, it saves time and resources. The records also provide evidence of the accountability in the hospitals for its actions, and they form a key source of data for medical research, statistical reports, and health information systems [10].

Numerous previous studies [6-8] address the context of building IoT healthcare systems. The previous research describes the connected health model and the emerging related technologies towards the distributed health information sharing. They address the problem of establishing standard communication in IoT healthcare systems, focusing on related communication protocol evaluation [7]. Similarly, other previous researchers provide a representation of an IoT healthcare system. It is the general description with a special presentation of its related infrastructure. They tackle the security issues with a presentation of potential vulnerability by the major cyber-attack [6]. Then, some researchers present a general-purpose healthcare system that interacts with a cloud environment. They propose a healthcare system architecture by focusing on how to maintain system performance in a distributed context. This performance is ensured by implementing a unified data flow with scheduling algorithms to ensure the fair distribution of system resources [8]. However, the software patient service and its related interfaces and design do not address the related works.

\section{B. Internet of Things (IoT) Background}

The IoT concepts are drawn by its roots from the founder concept of "smart devices". This idea, which started in the 90s, is the basis behind the nowadays evolution of IoT. However, the first establishment of this concept is made in the last decade of the previous century [3]. Many common factors have led to the great evolution of IoT, such as the Internet growth, network evolution (3G, 4G, LTE, and 5G), accessibility of technology, and the technology integration with daily lives [11].

However, the concepts have been raised momentum at the beginning of the current century, basically, with the Internet Protocol for Smart Objects (IPSO) alliance formed by around fifty-member big technology companies such as Cisco, Intel, Sap, Sun, Google, and others. There is a growth report of smart devices by Cisco Internet Business Solutions Group (IBSG) in the same year. It depicts that the number of connected devices worldwide has overtaken the human population before 2010. It also predicts that by 2020, it will reach the fifty-billion connected devices. The statistic is presented in Fig. 1. 
Cite this article as: M. N. Miladi, A. R. Hasan, M. A. Ghobiri, O. Nasr, and A. Abelmotlab, "Towards an Internet of Things for Health Centers in the Southern Saudi Arabia Region", CommIT (Communication \& Information Technology) Journal 15(1), 1-7, 2021.

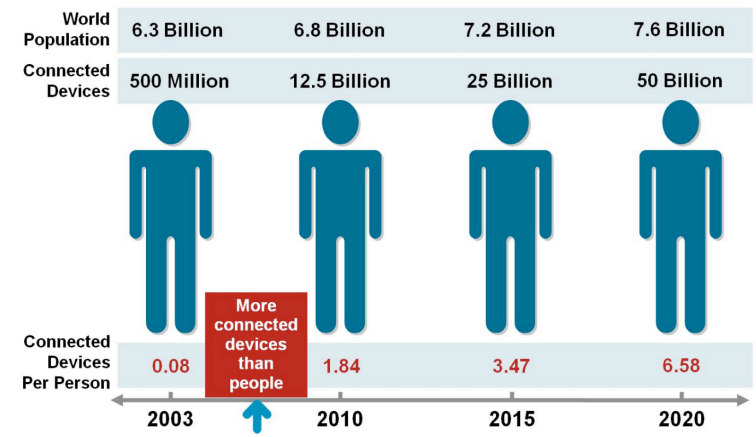

Fig. 1. The evolution of connected devices per person over past years.

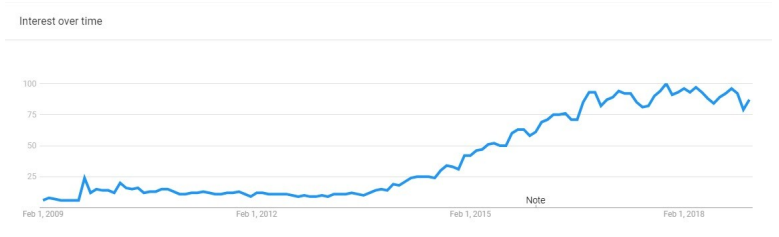

Fig. 2. The Internet of Things interest over past years.

Nowadays, IoT is one of the most relevant fields in web searches worldwide. The IoT interest (delivered by Google) is depicted in Fig. 2. It represents the IoT interest over the last ten years and exhibits the high evolution of IoT interest worldwide, especially in the last five years. Many honorable consultant offices predict IoT will lead the innovative emerging business process and models. Some of them even go further by saying that innovative sectors will be based on IoT. Hence, current companies based on the traditional business model may be driven out of business [12].

Nowadays, IoT is one of the most relevant fields in web searches worldwide. The IoT interest (delivered by Google) is depicted in Fig. 2. It represents the IoT interest over the last ten years and exhibits the high evolution of IoT interest worldwide, especially in the last five years. Many honorable consultant offices predict IoT will lead the innovative emerging business process and models. Some of them even go further by saying that innovative sectors will be based on IoT. Hence, current companies based on the traditional business model may be driven out of business [12].

\section{Responsibilities of the Health Centers towards the Patients}

Some general responsibilities in health centers include:

1) Be fully aware of the patients' rights and responsibilities of health centers

2) Be the best possible healthcare
3) Identify the identity and professional character of the working staff and the responsible doctor for the patients' treatment

4) Obtain a comprehensive explanation and information about diagnosing patients' condition

5) Protect the confidentiality of medical data and information

6) Obtain copies of diagnostic papers or medical reports upon request to receive treatment in a healthy and safe environment.

\section{RESEARCH MethoD}

\section{A. Proposed System}

The patient file management system is designed for health centers in the village. It is to replace their existing manual paper-based system. The new system is comprehensive by integrating the designed information systems to manage the information of patients and service processes. Therefore, the system must be user-friendly, simple, fast, and cost-effective. It can deal with collecting information regarding the patients, departments, doctors, diagnosis details, and others.

The main function of the system is to register and store the details regarding the patients and doctors, retrieve these details when they are required, and change these details meaningfully. The system input contains details of patients and diagnoses. Meanwhile, the system output is to get these details on the screen. The system can be entered using a username and password. It is accessible by an administrator, doctor, or receptionist. Only they can add data into the database. Moreover, the data can be retrieved quickly to reduce the currently required time and resources for such tasks. Also, patient data are well protected for personal use and make the data processing very fast.

\section{B. Analysis Diagram}

The whole analysis diagrams are realized through the Visual Paradigm tool suite. The analysis of the proposed system is achieved through the Use Case diagram as depicted in Fig. 3. It shows the various system actors, including the administrator, the receptionist, and the doctor. The researchers model the basic units of useful functions, such as managing patient files, chronic diseases, and doctors. The Use Case diagram also presents the interaction in the system with the various users. Afterward, the structure of the proposed system is described using a Class diagram in Fig. 4. It mentions the basic structure of the proposed system. The classes of staff, administrator, receptionist, and doctor provide the basic actors' structure, including appointments and consults. It establishes the basic 
Cite this article as: M. N. Miladi, A. R. Hasan, M. A. Ghobiri, O. Nasr, and A. Abelmotlab, "Towards an Internet of Things for Health Centers in the Southern Saudi Arabia Region", CommIT (Communication \& Information Technology) Journal 15(1), 1-7, 2021.

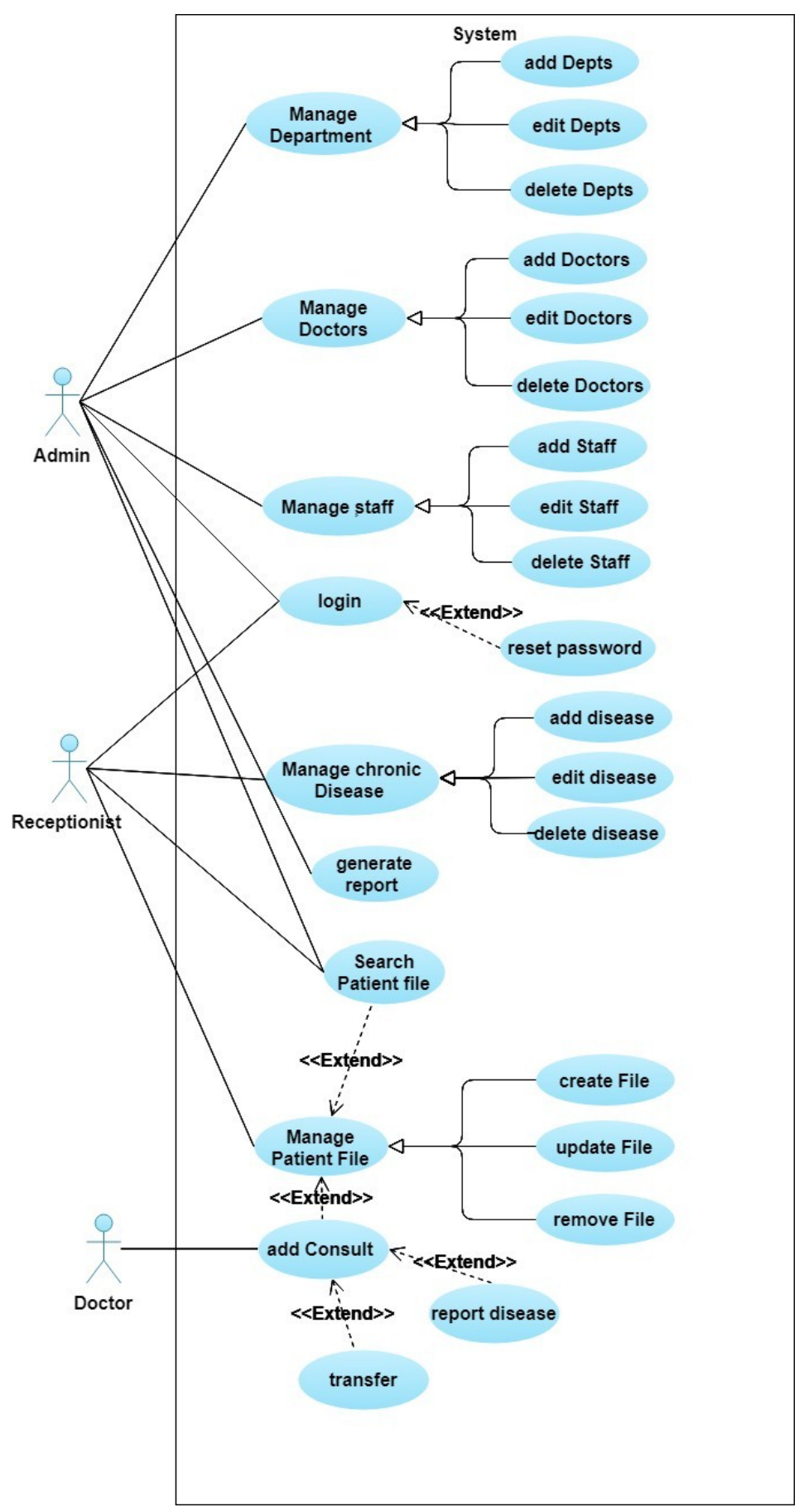

Fig. 3. The Use Case diagram.

structure of the system services. Figure 4 also describes the required class relations.

\section{RESULTS AND Discussion}

The proposed healthcare solution offers several results. Those are described in two parts. The first one describes the basic interface in the system. The second one focuses on the major system contributions, including functional and nonfunctional services.

\section{A. Interface Design}

The dashboard is where the administrator can control the system and user data. The administrator will be 
Cite this article as: M. N. Miladi, A. R. Hasan, M. A. Ghobiri, O. Nasr, and A. Abelmotlab, "Towards an Internet of Things for Health Centers in the Southern Saudi Arabia Region", CommIT (Communication \& Information Technology) Journal 15(1), 1-7, 2021.

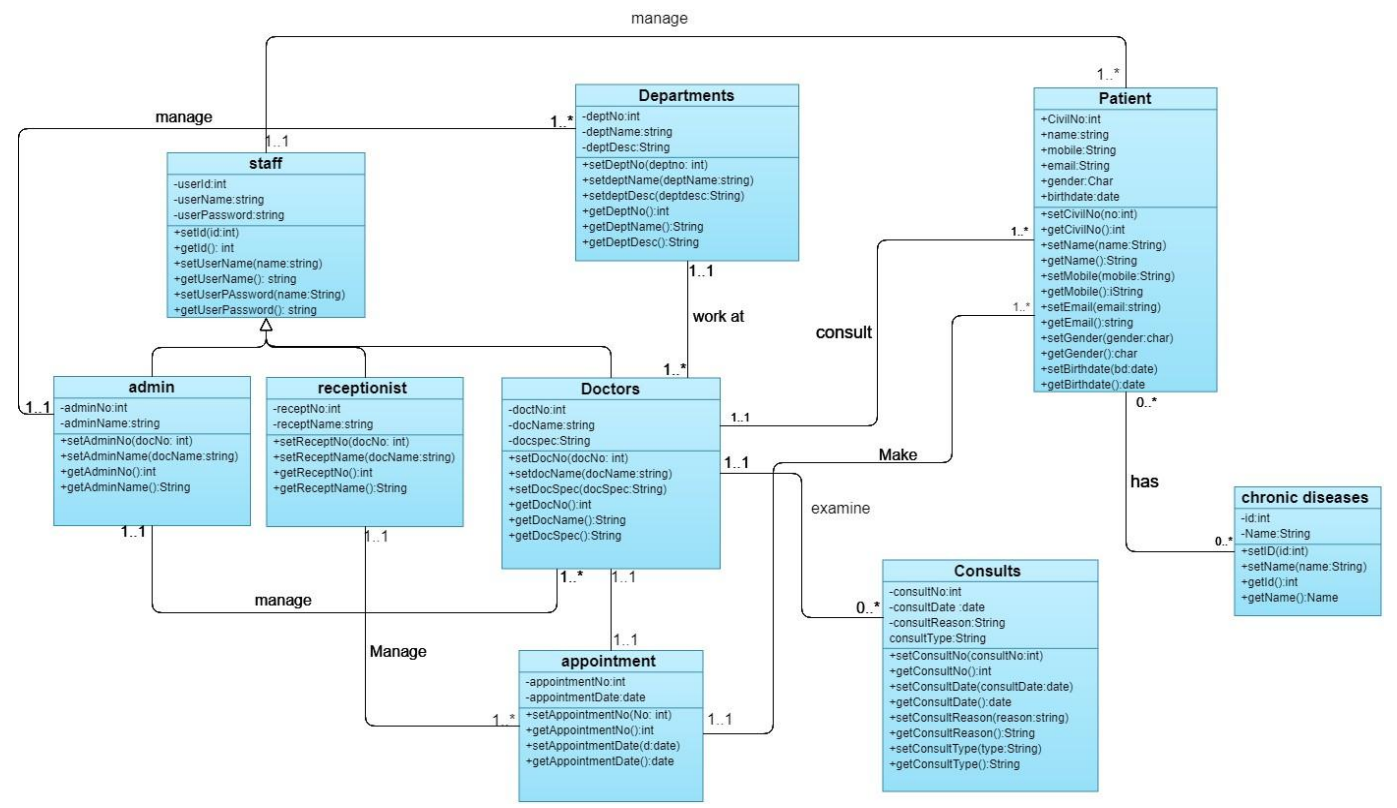

Fig. 4. The Class diagram.

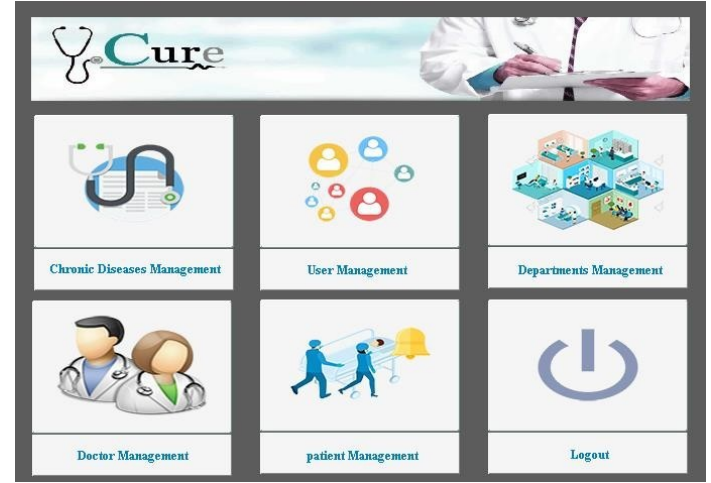

Fig. 5. The administrator's panel form.

redirected to this form as depicted in Fig. 5 after proving his or her identity in the login form. The administrator has the privilege to manage information of department, chronic disease, doctor and search the needed patient files. In addition, the most important role for the administrator is to manage other users in the system. The administrator can create, update, or remove users, and manage the diseases, as shown in Fig. 6. The service in Fig. 7 allows the administrator or receptionist to find and retrieve patient files. The related information like name of the patient, gender, mobile number, email, birthdate, chronic disease, and consultation details given to patients will be obtained.

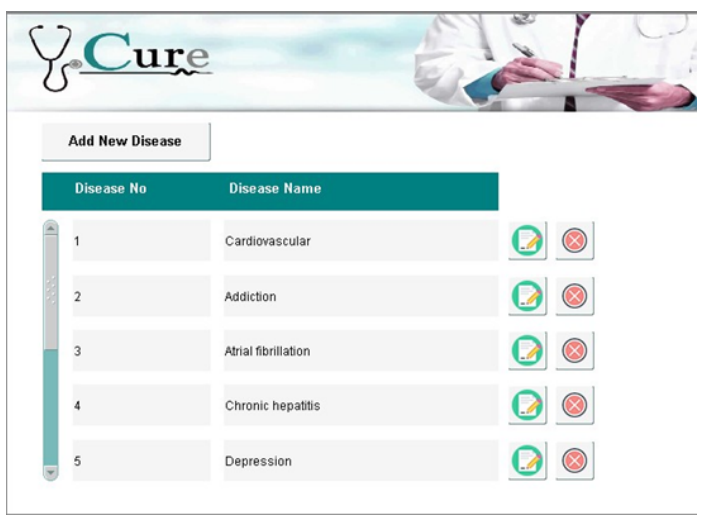

Fig. 6. Example of managing a chronic disease phase.

\section{B. Results}

The proposed system is automated patient file management by integrating hospital information and patient information into a central system database. It also provides receptionists with an easy way to book appointments according to patients' preferences. They can search and access patient information conveniently.

The result achieved in the research is a working patient file management application. It performs the stated requirements in this document. The system has three types of modules, includes administrator, doctor, and receptionist. The first module is for the administrator. It allows the administrator to manage departments of health centers, doctors, user accounts, 
Cite this article as: M. N. Miladi, A. R. Hasan, M. A. Ghobiri, O. Nasr, and A. Abelmotlab, "Towards an Internet of Things for Health Centers in the Southern Saudi Arabia Region", CommIT (Communication \& Information Technology) Journal 15(1), 1-7, 2021.

TABLE I

IMPLEMENTATION RESUlTS OF THE PROPOSED SYSTEM.

\begin{tabular}{|c|c|c|c|c|c|c|c|}
\hline Type of the user & Total & Factor & Very Satisfied & Somewhat Satisfied & Neutral & Somewhat Unsatisfied & Very Unsatisfied \\
\hline \multirow{2}{*}{ Doctors } & \multirow{2}{*}{8} & User Interface Design & 0 & 5 & 3 & 0 & 0 \\
\hline & & Usefulness & 1 & 6 & 1 & 0 & 0 \\
\hline \multirow{2}{*}{ Administrators } & \multirow{2}{*}{2} & User Interface Design & 1 & 1 & 0 & 0 & 0 \\
\hline & & Usefulness & 1 & 1 & 0 & 0 & 0 \\
\hline \multirow{2}{*}{ Receptionists } & \multirow{2}{*}{10} & User Interface Design & 4 & 2 & 2 & 1 & 1 \\
\hline & & Usefulness & 3 & 4 & 1 & 1 & 1 \\
\hline
\end{tabular}

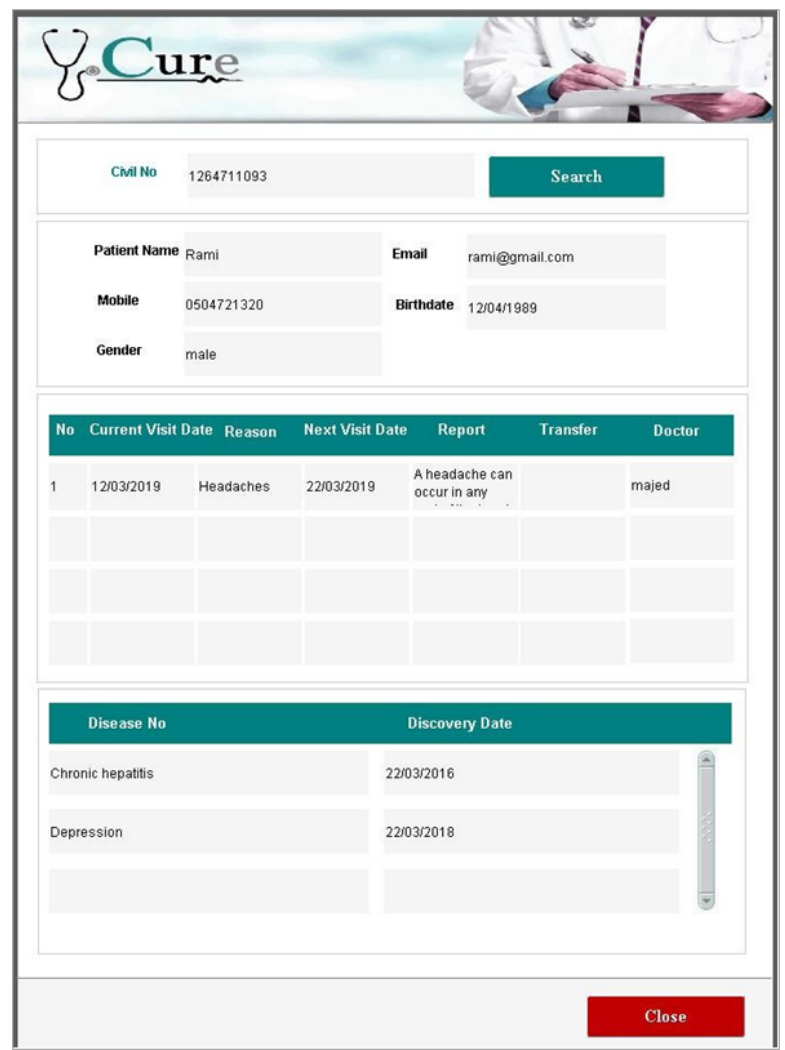

Fig. 7. Example of finding and retrieving patient information phase.

and chronic diseases and search patient files. The second module is for the receptionist. The receptionist can create patient files, add chronic diseases to patient files, make appointments for patients, search for patient files, and retrieve all information related to patients and appointments in the system. Finally, the doctors' module allows them to review all related appointments and add consultant records to patient files after each patient's visit.

After testing the system modules against some input, the researchers find that the software is user friendly. It is designed to be performed well, scalable, extensible, and highly available. The usability of the system is also tested. The results are shown in Table I. It focuses on two factors: the ergonomic aspect of the system through the satisfactory scale of the user interface design and the system usefulness using the same scale. The whole system users are involved in the usability study.

The proposed system ensures the privacy of the users' data. It secures its access with a dedicated service. An enhanced security module that can ensure system control in the case of integrating the proposed system with other health systems (government hospital system) is under process with an associated project to cover security vulnerability in IoT $[6,13,14]$. The medium-term objective is to provide an IoT-based system for healthcare in the southern Saudi Arabia region. It takes advantage of the enormous communication and network advances (establishment of the five-generation wireless network) and the continuous increase in communication devices. The integrated global solution is articulated on layered-based healthcare architecture. The proposed solution is included in the service layer part. Then, the security issue involves the control layer part.

\section{CONCLUSION}

The proposed solution provides a computerized version for patient file management in health centers. It will benefit the small health centers and the receptionists who currently depend on the manual system. It makes the entire process automated. The administrator can $\log$ in to manage information of departments, doctors, chronic diseases, and other users' accounts. The receptionist can also create, update, find, and access patient files in the system.

Future research of this system can add more features to accommodate other users, such as doctors, pharmacies, and nurses who can access patient files, retrieve needed information, and update files directly on needs. On the other hand, further research can improve an online portal to allow patients to register and manage their membership profile. This portal can provide 24hour access for patients to manage their appointments, retrieve the diagnosis results given by doctors, see laboratory analysis results, and more. Thus, the additional features will make the system more interactive and user 
Cite this article as: M. N. Miladi, A. R. Hasan, M. A. Ghobiri, O. Nasr, and A. Abelmotlab, "Towards an Internet of Things for Health Centers in the Southern Saudi Arabia Region", CommIT (Communication \& Information Technology) Journal 15(1), 1-7, 2021.

friendly. The system can fulfill each user's need in the best possible way.

\section{ACKNOWLEDGEMENT}

The research is supported by a grant from the King Khaled University. The authors are indebted to the Faculty of Business and Deanship of Scientific Research at King Khalid University for funding the research through General Research Project under grant number (175/39).

\section{REFERENCES}

[1] A. Alenezi, Z. AlMeraj, and P. Manuel, "Challenges of IoT based smart-government development," in $201821^{\text {st }}$ Saudi Computer Society National Computer Conference (NCC). Riyadh, Saudi Arabia: IEEE, April 25-26, 2018, pp. 1-6.

[2] V. Gazis, M. Goertz, M. Huber, A. Leonardi, K. Mathioudakis, A. Wiesmaier, and F. Zeiger, "Short paper: IoT: Challenges, projects, architectures," in $201518^{\text {th }}$ International Conference on Intelligence in Next Generation Networks. Paris, France: IEEE, Feb. 17-19, 2015, pp. 145-147.

[3] M. Bhayani, M. Patel, and C. Bhatt, "Internet of Things (IoT): In a way of smart world," in Proceedings of the International Congress on Information and Communication Technology. Springer, 2016, pp. 343-350.

[4] K. A. Delic, "On resilience of IoT systems: The Internet of Things," Ubiquity, vol. 2016, no. February, pp. 1-7, 2016.

[5] O. Olamide, E. Adedayo, and O. Abiodun, "Design and implementation of hospital management system using Java," IOSR Journal of Mobile Computing \& Application, vol. 2, no. 1, pp. 3236, 2015.

[6] A. Djenna and D. E. Saïdouni, "Cyber attacks classification in IoT-based-healthcare infrastructure," in $20182^{\text {nd }}$ Cyber Security in Networking Conference (CSNet). Paris, France: IEEE, Oct. 24-26, 2018, pp. 1-4.

[7] K. Karamitsios, T. Orphanoudakis, and T. Dagiuklas, "Evaluation of IoT-based distributed health management systems," in Proceedings of the $20^{\text {th }}$ Pan-Hellenic Conference on Informatics, 2016, pp. 1-6.

[8] Y. Zhang, Y. Sun, R. Jin, K. Lin, and W. Liu, "High-performance isolation computing technology for smart IoT healthcare in cloud environments," IEEE Internet of Things Journal, 2021.

[9] M. G. K. Ripan, "Online hospital management system," Ph.D. dissertation, Department of Computer Science and Engineering, BRAC University, 2017.
[10] P. K. Bishwakarma, M. Upreti, and R. K. Yadav. (2014) A proposal on hospital management system. [Online]. Available: https://bit.ly/3kE1oYb

[11] T. Orehovački, D. P. Vukovac, M. Džeko, and Z. Stapić, "Evaluating relevant UX dimensions with respect to IoT ecosystem intended for students' activities tracking and success prediction," in International Conference on Learning and Collaboration Technologies. Las Vegas, NV, USA: Springer, July 15-20, 2018, pp. 279-293.

[12] R. Van Kranenburg and A. Bassi, "IoT challenges," Communications in Mobile Computing, vol. 1, no. 1, pp. 1-5, 2012.

[13] E. Bertino, K. K. R. Choo, D. Georgakopolous, and S. Nepal, "Internet of Things (IoT) smart and secure service delivery," ACM Transactions on Internet Technology, vol. 16, no. 4, pp. 1-7, 2016.

[14] R. De Michele and M. Furini, "IoT healthcare: Benefits, issues and challenges," in Proceedings of the $5^{\text {th }}$ EAI International Conference on Smart Objects and Technologies for Social Good, 2019, pp. 160-164. 